\title{
Multilayered Fibroblasts Constructed by Accelerated Cellular Self-Assembly and Applications for Regenerative Medicine
}

\author{
Paninee Chetprayoon ${ }^{1}$, Sasitorn Aueviriyavit ${ }^{1}$, Arisara Prateep ${ }^{1}$, Sirima Boonjing ${ }^{2}$ \\ and Rawiwan Maniratanachotel \\ ${ }^{1}$ Nano Safety and Risk Assessment Laboratory, National Nanotechnology Center (NANOTEC), National Science and Technology Development Agency \\ (NSTDA), Pathum Thani, Thailand; ${ }^{2}$ Department of Pharmacology and Physiology, Faculty of Pharmaceutical Sciences, Chulalongkorn University, \\ Bangkok, Thailand
}

\begin{abstract}
Regenerative medicine research requires animal experiments to evaluate treatment effects. According to the 3 Rs principles, alternative models have been developed and utilized to evaluate the efficacy and safety of new products. Three-dimensional (3D) cell cultures have been recognized for their relevant structures and biological functions that are akin to native tissues. They can better represent in vivo conditions than two-dimensional (2D) cell cultures. Herein, we present a fast and simple technique for the construction of 3D dermal fibroblasts (3D-DFs) without exogenous scaffolds. The 3D-DFs can be obtained within 3 days by seeding DFs at a level that exceeds their confluent density and culturing them in the presence of ascorbic acid. The 3D-DFs have a compact, multilayer structure as revealed by histology and their collagen content is drastically increased compared to the monolayer. The 3D-DF-derived extracellular matrix can serve for 3D culturing of other cells. A gap closure assay was performed with the 3D-DFs to represent a 3D-wounded dermal model. Interestingly, the multilayered structure of the 3D-DFs could be regenerated after wounding even when cultured in the absence of ascorbic acid. Moreover, skin grafting using the 3D-DFs was demonstrated in vitro using wounded in vitro human full-thickness skin models. The 3D-DFs will be potentially useful for regenerative medicine and as tissue models for in vitro studies.
\end{abstract}

\section{Introduction}

In vivo tissues are composed of a complex system of three-dimensional (3D) cellular multilayers of various types of cells and extracellular matrix (ECM). Animals are usually used as in vivo models for testing the toxicity and effectiveness of new products, including chemicals, drugs, consumer products and medical devices. However, animals cannot represent all human conditions since they are genetically different from humans.

Alternatives to animal experiments have been developed that include in vitro and ex vivo approaches. Since two-dimensional (2D) in vitro models composed of a monolayer of cells poorly reflect the in vivo situation, recent research has realized the significance of $3 \mathrm{D}$ cultures over $2 \mathrm{D}$ cultures due to their more relevant structure and cellular functions compared to native tissues (Griffith and Swartz, 2006; Bokhari et al., 2007; Onoe et al., 2013; Knight and Przyborski, 2015). However, methods for constructing 3D tissues are typically a complex process and still remain challenging. In general, primary cells cultured on a substrate propagate along a 2D plane since, unlike cancerous or transformed cells, they do not form a 3D multilayer once they become confluent due to contact inhibition (Takai et al., 2008). Scaffolds derived from various biomaterials can be applied for the construction of 3D tissues (Bokhari et al., 2007; Lee et al., 2009; Kim et al., 2015). Nevertheless, the presence of these scaffold materials can disturb the tissue remodeling and functions (Anderson et al., 2008; Alaribe et al., 2016), and can induce undesired foreign body reactions following implantation (Anderson
Received April 3, 2019; Accepted September 30, 2019;

Epub October 2, 2019; ( ) The Authors, 2019

ALTEX 37(1), 075-084. doi:10.14573/altex.1904032

Correspondence: Rawiwan Maniratanachote

National Science and Technology Development Agency (NSTDA)

111 Innovation Cluster 2

Thailand Science Park, Phahonyothin Rd.

Khlong Nueng, Khlong Luang

Pathum Thani 12120 , Thailand

(rawiwan.man@nstda.or.th)
This is an Open Access article distributed under the terms of the Creative Commons Attribution 4.0 International license (http://creativecommons.org/licenses/by/4.0/) which permits unrestricted use, distribution and reproduction in any medium, provided the original work is appropriately cited. 
A
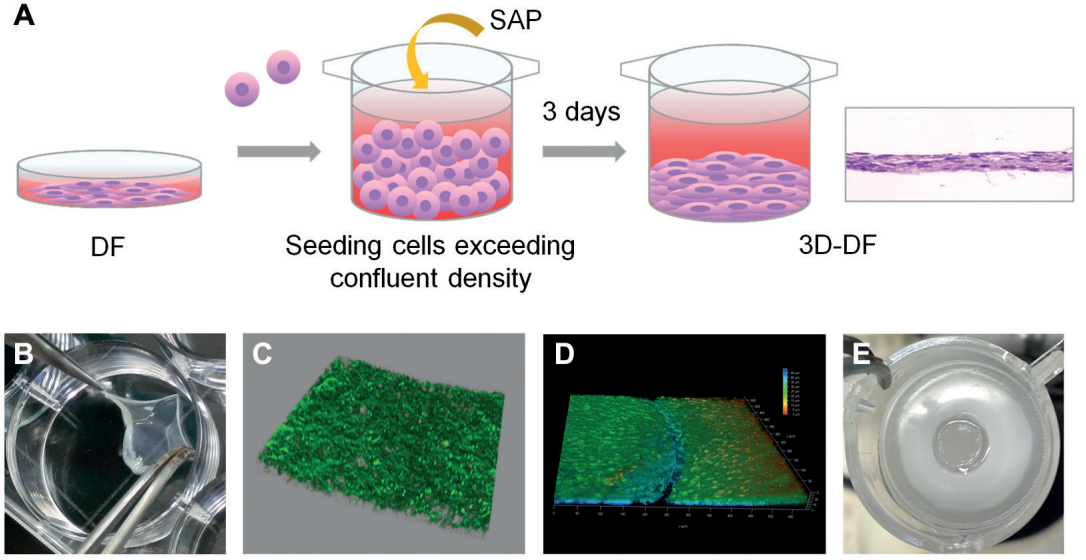

Fibroblast sheet

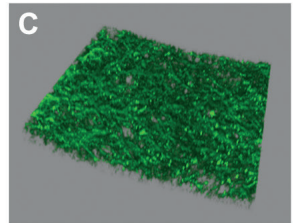

3D-DF-derived ECM

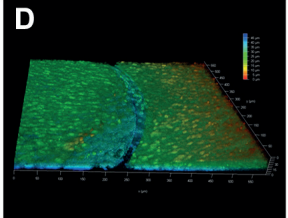

3D wound healing

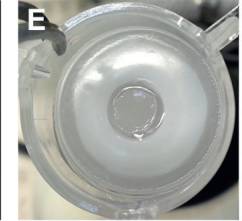

In vitro dermal graft
Fig. 1: Construction of 3D-DFs by accelerated cellular self-assembly and their applications in this study (A) Schematic illustration of the construction of the 3D-DFs. The DFs were seeded at an overconfluent density and cultured in SAP-containing medium for 3 days, resulting in multilayered DFs. (B) Harvesting of an intact sheet of 3D-DF after 3 days of culture. (C) 3D-DF-derived ECM after decellularization. (D) 3D wound healing study using 3D-DFs. (E) In vitro dermal graft of 3D-DFs on a full thickness skin model. et al., 2008; Gerullis et al., 2014; Dondossola et al., 2017). Furthermore, exogenous materials can interfere with the transport of oxygen, nutrients and some large molecules in the cell culture, such that highly interconnected porous scaffolds are usually desired (Ramanujan et al., 2002; Loh and Choong, 2013). Thus, a method to construct 3D cell cultures without the use of exogenous materials is highly desirable.

A cellular self-assembly approach can enable the construction of 3D tissues without using exogenous materials. The concept of tissue engineering by self-assembly is based on the ability of cells to produce ECM such that living tissues such as skin (Jean et al., 2009, 2011) and vascular walls (L'Heureux et al., 1998; Peck et al., 2011) can be constructed under appropriate culturing conditions without synthetic materials. Fibroblasts, the main components of connective tissues, can synthesize ECM proteins, particularly collagen, that are important for maintaining the structural integrity of tissues. Ascorbic acid and its derivatives are known to stimulate collagen production in fibroblasts (Murad et al., 1983; Geesin et al., 1988; Chung et al., 1997) as ascorbic acid acts as a co-factor in the hydroxylation of proline to hydroxyproline, the characteristic amino acid of collagen, which stabilizes the collagen triple helices by incorporating them into collagen peptides (Barnes, 1975).

When fibroblasts were cultured in a medium containing ascorbic acid for more than two weeks, they formed multilayered tissues (Hata and Senoo, 1989; Ohgoda et al., 1998), and a sheet of fibroblast tissue could be harvested after 5 weeks of culture (Michel et al., 1999; Jean et al., 2009). Thus, although multilayered tissues can be obtained by culturing cells that produce an ECM under appropriate conditions, such approaches take a long time.

Herein, we demonstrate that 3D multilayered fibroblasts can be successfully constructed within 2-3 days using the self-assembly approach. This acceleration of the cellular self-assembly pro- cess was achieved by seeding dermal fibroblasts (DFs) at a high density and by adding ascorbic acid to stimulate collagen production. The 3D structure was found to significantly modulate collagen production in the presence of ascorbic acid. We further show potential applications of the resulting 3D-DFs in tissue engineering (Fig. 1). The ECM could be stripped of fibroblasts by decellularization and used as a scaffold to support the 3D culture of fresh fibroblasts, instead of using synthetic materials or an animal-derived matrix. Furthermore, the multilayered structure of the 3D-DFs could be regenerated after wounding. Finally, the 3D-DFs were grafted onto a wounded in vitro commercial full-thickness human skin model. Thus, the 3D-DFs are potentially useful for in vitro studies and medical applications.

\section{Materials and methods}

\section{Cell culture}

Normal human DFs (ATCC, Manassas, VA, USA) at passages 9-15 were used. They were cultured in complete medium (Dulbecco's Modified Eagle Medium containing 10\% (v/v) fetal bovine serum and $2 \mathrm{mM}$ L-alanyl-L-glutamine) (Invitrogen, Carlsbad, CA, USA) at $37^{\circ} \mathrm{C}$ in a humidified atmosphere with $5 \%(\mathrm{v} / \mathrm{v}) \mathrm{CO}_{2}$.

\section{Construction of $3 D-D F s$}

The DFs were seeded onto a $6.5 \mathrm{~mm}$ culture Transwell (Corning, NY, USA, surface area of $0.33 \mathrm{~cm}^{2}$ ) at a cell density of $2 \times 10^{5}$ cells $/ \mathrm{cm}^{2}$ for the construction of a single cell layer (named 1L) and cultured in a $24-$ well plate. The cell seeding densities used for construction of 3D-DFs with different numbers of cellular layers are shown in Table 1. The 3D-DFs were constructed at a $5 \mathrm{~L}$ thickness by seeding DFs at a density 
Tab. 1: Cell seeding density for construction of 3D-DFs with different cellular layers used in this study

The number of cellular layers $(L)$ is reflected in the fold number of cell seeding density.

\begin{tabular}{|l|l|}
\hline Number of cell layer(s) (L) & Cell seeding density $\left(\right.$ cells $\left./ \mathbf{c m}^{2}\right)$ \\
\hline 1 & $2 \times 10^{5}$ \\
\hline 2 & $4 \times 10^{5}$ \\
\hline 3 & $6 \times 10^{5}$ \\
\hline 5 & $1 \times 10^{6}$ \\
\hline 7 & $1.4 \times 10^{6}$ \\
\hline 10 & $2 \times 10^{6}$ \\
\hline
\end{tabular}

of $1 \times 10^{6}$ cells $/ \mathrm{cm}^{2}$ (five-fold the $1 \mathrm{~L}$ cell density) and cultured for 3 days, except where otherwise stated. After seeding, the cells were cultured in complete medium supplemented with $0.1 \mathrm{mM}$ 2-phospho-L-ascorbic acid trisodium salt (SAP; Sigma-Aldrich, St. Louis, MO, USA). The volume of culture medium in the Transwell and in the well was $0.3 \mathrm{~mL}$ and $1.5 \mathrm{~mL}$, respectively. After $24 \mathrm{~h}$ culture, an additional $0.5 \mathrm{~mL}$ of medium was added to the culture.

\section{Determination of collagen content}

The total collagen content of the 3D-DFs was evaluated and compared with that of 2D (monolayer) DFs. The 3D multilayer and the 2D monolayer were constructed by seeding $3.35 \times 10^{5}$ cells on the different surface areas of a $6.5 \mathrm{~mm}$ Transwell $\left(0.33 \mathrm{~cm}^{2}\right.$ surface area, equivalent to $1 \times 10^{6}$ cells $/ \mathrm{cm}^{2}$ (5L cell density)) and a 12 -well plate $\left(3.8 \mathrm{~cm}^{2}\right.$ surface area, equivalent to $0.9 \times 10^{5}$ cells/ $\mathrm{cm}^{2}$ ) for the 3D multilayer and the 2D monolayer, respectively. The DFs were cultured in culture medium without SAP or with $0.1 \mathrm{mM}$ SAP.

The collagen content was determined by hydroxyproline assay following previously described methods (Lee et al., 2011) with some modifications. Briefly, all samples were harvested from the culture insert by trypsinization. For the 3D-multilayer cultured in culture medium with SAP, a pipette tip was used to scratch and peel the tissue off the membrane. The collected cells were then suspended in $6 \mathrm{M}$ hydrochloric acid solution at $95^{\circ} \mathrm{C}$ overnight $(22 \pm 3 \mathrm{~h})$. The hydrolyzed samples were dried in a vacuum concentrator and then reacted with chloramine-T and p-dimethylaminobenzaldehyde (Sigma-Aldrich). The absorbance was measured using a microplate reader at a wavelength of $555 \mathrm{~nm}$ (Molecular Devices, San Jose, CA, USA). Bovine type I collagen (BD Biosciences, San Jose, CA, USA) was used as a standard.

For analysis of type I collagen in the culture medium, the medium was collected on day 3 and analyzed using a human pro-collagen I alpha SimpleStep enzyme-linked immunosorbent assay kit (Cat. ab210966, Abcam, Cambridge, UK) according to the manufacturer's protocol.

Total cellular protein assay

Total cellular protein content, which is a representative measure of cell number, was quantified. Cells were lysed with Nonidet P-40 (NP-40) lysis buffer (1\% (v/v) NP-40, $50 \mathrm{mM}$ Tris-HCl, $150 \mathrm{mM} \mathrm{NaCl}, 1 \mathrm{mM}$ ethylenediaminetetraacetic acid (EDTA), $100 \mathrm{mM} \mathrm{NaF}, 0.2 \mathrm{mM} \mathrm{Na}_{3} \mathrm{VO}_{4}, \mathrm{pH} 8.0$ ), collected in microtubes and disrupted using a Vibra-Cell ${ }^{\mathrm{TM}}$ ultrasonic processor (SONICS, Newton, CA, USA). After centrifugation at 20,900 g, the supernatant was harvested and total protein content was determined by the Lowry method using a $\mathrm{DC}^{\mathrm{TM}}$ protein assay (BioRad, Hercules, CA, USA). Briefly, $5 \mu \mathrm{L}$ sample was added to a 96-well plate, followed by $25 \mu \mathrm{L}$ and $200 \mu \mathrm{L}$ of reagents $\mathrm{A}$ and $\mathrm{B}$, respectively, and mixed well. After $15 \mathrm{~min}$ of incubation at room temperature, the absorbance was read at $750 \mathrm{~nm}$.

\section{Decellularization of the $3 D-D F s$}

The 3D-DFs were seeded at 7L cell density and were decellularized after 3 days following a previously published method (Xing et al., 2015). Briefly, a first decellularization solution ( $1 \mathrm{M} \mathrm{NaCl}, 10 \mathrm{mM}$ Tris and $5 \mathrm{mM}$ EDTA) was applied to the 3D-DFs for $1 \mathrm{~h}$, followed by immersion in a second decellularization solution $(0.05 \%(\mathrm{w} / \mathrm{v})$ sodium dodecyl sulfate (SDS), $10 \mathrm{mM}$ Tris and $25 \mathrm{mM}$ EDTA) for $30 \mathrm{~min}$. Residual decellularized 3D-DFs were washed with phosphate buffered saline (PBS).

In the recellularization experiments, the decellularized 3DDFs were reseeded with DFs at 5L density and cultured for 1 day in culture medium without SAP. Decellularized and the recellularized tissues were fixed with $4 \%$ paraformaldehyde (PFA) and washed three times with PBS before immunostaining.

\section{Gap closure assay of $3 D-D F s$}

After 3 days of culture, wounds were made aseptically on 3D-DFs using a $1 \mathrm{~mm}$-diameter punch (Acuderm, Fort Lauderdale, FL, USA). The punched tissue was carefully removed using forceps. The wounded 3D-DFs were then cultured in fresh culture medium without SAP for 10 days, replacing the culture medium with fresh medium three times a week.

Use of the 3D-DF as a dermal substitute on wounded skin models $3 \mathrm{D}-\mathrm{DFs}$ at $10 \mathrm{~L}$ cell density were constructed. Rho kinase inhibitor Y-27632 dihydrochloride (Millipore, Temecula, CA, USA) was added to the $3 \mathrm{D}$-DFs during the 3 days of culture to prevent tissue contraction (Gribova et al., 2016) when removing the membrane from the Transwell. The Transwell membranes containing the 3D-DFs were cut from the Transwell cup using a 6-mm punch to prepare the 3D-DF grafts. Full thickness human in vitro skin models (FTMs), consisting of dermis and epidermis (EpiSkin ${ }^{\mathrm{TM}}$, Lyon, France), were used to demonstrate dermal grafting in vitro. Full-thickness wounds were made at the center of the FTMs using an 8-mm punch. Dermis and epidermis in the cut area were removed by forceps, leaving the wounded area. The 3D-DF graft was then placed into the wounded area of the FTMs.

In another experiment, $50 \mu \mathrm{L}$ of medium containing $1 \times 10^{5} \mathrm{DFs}$ were seeded onto the wounded area of the FTMs, while in the control the wounded FTMs were cultured in complete medium alone without grafting. 
A

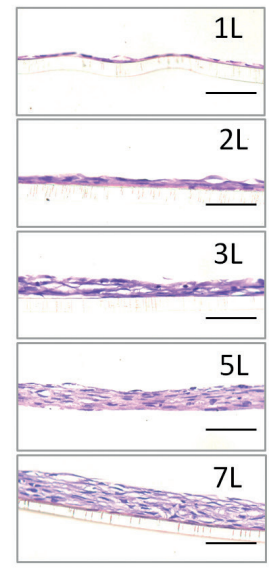

B

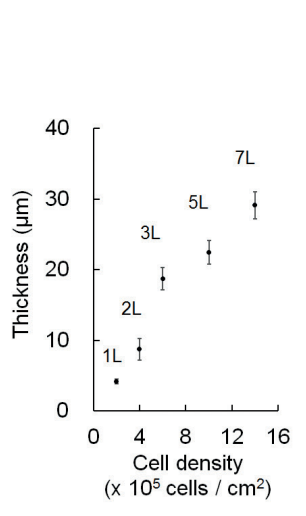

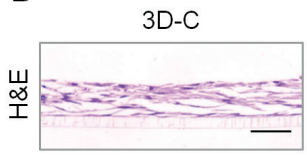

3D-C

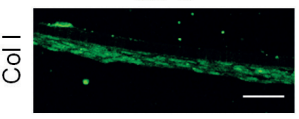

3D-C

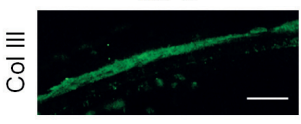

$3 \mathrm{D}-\mathrm{C}$

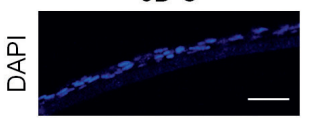

3D-S

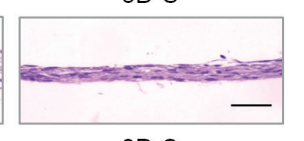

3D-S

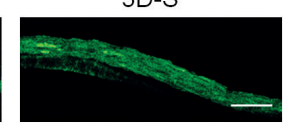

3D-S

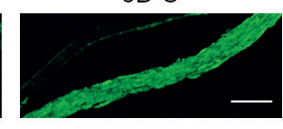

3D-S

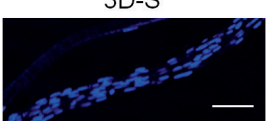

Fig. 2: Structure of the 3D-DFs constructed by accelerated cellular self-assembly

(A) Histological structure and thickness of the 3D-DFs cultured in the presence of $0.1 \mathrm{mM}$ SAP for 3 days at different cell seeding densities. Note that $1 \mathrm{~L}$ refers to the cell seeding density that resulted in a monolayer of cells. (B) Immunostaining of 3D-DFs cultured in medium without SAP (3D-C) or with 0.1 mM SAP (3D-S) at $5 \mathrm{~L}$ cell density for 3 days. Cryosections of 3D-DFs were immunostained with type I collagen (green), type III collagen (green) and DAPI (blue). Images were taken at a similar laser intensity. Scale bars are $50 \mu \mathrm{m}$.

\section{Histological images}

The 3D-DFs were fixed in 4\% PFA and then processed for histological sections and stained using hematoxylin \& eosin (H\&E) or immunofluorescence. The tissue thickness was estimated at five points of each $H \& E$ section using Image ${ }^{1}$ software.

\section{Immunofluorescence staining}

After fixation, the 3D-DFs were immunostained or processed for cryosections before immunostaining. Samples were permeabilized with $0.2 \%(\mathrm{v} / \mathrm{v})$ Triton X-100 and blocked with $1 \%$ $(\mathrm{w} / \mathrm{v})$ bovine serum albumin (BSA). Samples were incubated in $0.1 \%(\mathrm{w} / \mathrm{v})$ BSA containing primary antibody for $1 \mathrm{~h}$, washed in PBS, and stained with secondary antibody in $0.1 \%(\mathrm{w} / \mathrm{v})$ BSA for $30 \mathrm{~min}$. For anti-fibrillin-1 (Cat. MAB1919, Millipore), tissues were incubated with primary antibody at $4^{\circ} \mathrm{C}$ overnight. Monoclonal anti-human type I collagen (Cat. ab90395, Abcam) and monoclonal anti-human type III collagen antibodies (Cat. MAB2515, Millipore) were used at a 1:500 dilution. Alexa 568-conjugated phalloidin (Cat. A12380, Invitrogen), monoclonal anti-Ki-67 (Cat. MAB4190, Millipore) and anti-fibrillin-1 were used at a 1:100 dilution. Alexa 488-conjugated secondary antibody (Cat. ab150117, Abcam) was used at 1:200 dilution. Samples were stained with 4',6-diamidino-2-phenylindole, dihydrochloride (DAPI; Sigma-Aldrich) at 1:1000 dilution for 20 min. Samples were imaged with confocal laser scanning microscopy (CLSM; TCS SP8, Leica Microsystems, Wetzlar, Germany).

\section{Statistical analysis}

The numerical data are shown as the mean \pm SD derived from three independent experiments. Statistical significance of dif- ferences between means was assessed by ANOVA followed by Tukey's post-hoc test, accepting significance at the $p<0.05$ level.

\section{Results}

\subsection{Determination of the optimal SAP concentration and culture period for construction of the 3D-DFs}

DFs were seeded at 5L cell density to produce 3D-DFs and cultured for 3 days. The most effective concentration of SAP for obtaining the multilayered structure was evaluated across a $0-0.2$ $\mathrm{mM}$ range. Cross-sectional images of $\mathrm{H} \& \mathrm{E}$ stained DFs cultured without SAP revealed a loose structure, where most of the cells did not adhere to each other (Fig. S1 $\mathrm{A}^{2}$ ). Adding SAP at a concentration of at least $0.05 \mathrm{mM}$ led to the formation of a multilayered tissue. Increasing the concentration of SAP above $0.1 \mathrm{mM}$ did not further improve the histological structure, so the concentration of $0.1 \mathrm{mM} \mathrm{SAP}$ was selected for further studies.

Next, a suitable culture period for construction of the 3D-DFs was investigated. The DFs were cultured in the presence of 0.1 $\mathrm{mM}$ SAP for 1 to 7 days (Fig. S1B ${ }^{1}$ ). An intact multilayered structure was obtained 2 days after seeding. The tissue structure appeared even denser 3 days after seeding and became less dense 7 days after seeding. The expression of fibrillin-1, a microfibril component, and ECM glycoproteins also decreased between day 3 and day 7 (Fig. S8 ${ }^{1}$ ), suggesting disintegration of ECM components over time. The collagen level of 3D-DFs increased threefold from day 1 to day 3 (Fig. S2 ${ }^{1}$ ). Accordingly, a condition of $0.1 \mathrm{mM}$ SAP and a culture time of 3 days was selected.

\footnotetext{
1 https://imagej.nih.gov/ij/

2 doi:10.14573/altex.1904032s
} 
A

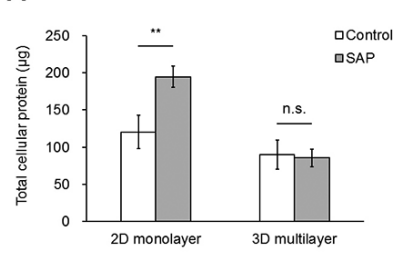

B

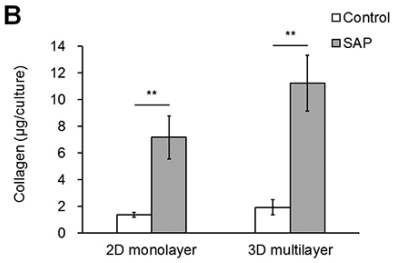

C

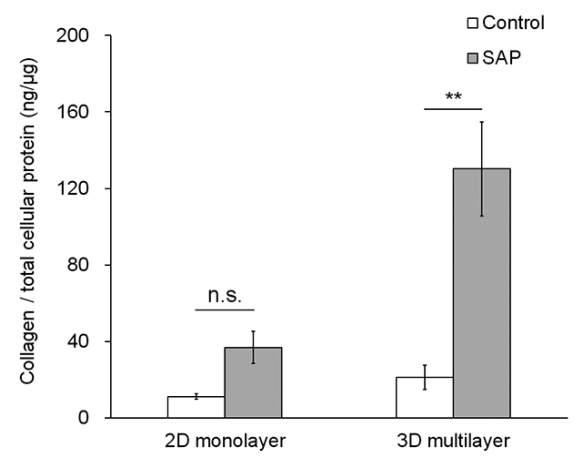

Fig. 3: Enhanced effects of SAP on collagen production in the 3D cellular structure

Total (A) cellular protein content in the cell lysate, $(\mathrm{B})$ collagen level in the cell cultures (measured as hydroxyproline) and (C) collagen level normalized to total cellular protein. The same number of cells were seeded in the $2 \mathrm{D}$ monolayer and the 3D multilayer. Data are shown as mean $\pm \mathrm{SD}, \mathrm{n}=3$. ${ }^{*} p<0.05 ;{ }^{* *} p<0.01$; n.s., not significant.

\subsection{Structure of the constructed 3D-DFs}

The thickness of the 3D-DFs was adjusted by altering the seeding density (Fig. 2A). A seeding density of $2 \times 10^{5}$ cells $/ \mathrm{cm}^{2}$ yielded a monolayer of cells (1L). The thickness of the DF tissues increased proportionally to the cell seeding density. However, exceeding a 10L cell density occasionally resulted in aggregation of cells (data not shown).

To construct 3D-DFs thicker than 10L, we constructed 3D-DF by repeated cell seeding. A 10L 3D-DF was constructed first over three days, followed by reseeding 10L cell density onto the established tissue and culturing for two further days. As a result, we could double the thickness of the tissue (Fig. S3 ${ }^{1}$ ). However, the obtained tissue showed a less homogeneous structure and non-uniform thickness.

Expression of type I and type III collagen, the major components of dermal ECM, was visualized by immunostaining cryosections of the 3D multilayers grown in culture medium with or without SAP, as shown in Figure 2B. Both types of collagen were expressed throughout the SAP-treated sections.

\subsection{Effects of SAP and the 3D cellular structure on collagen production}

2D monolayer and 3D multilayer DFs, initiated by seeding the same number of DFs on different surface areas in the presence or absence of SAP, were compared in order to investigate whether multilayer structure affects collagen production. The $2 \mathrm{D}$ monolayer showed a 1.6-fold increase in total cellular protein content when cultured in the presence of SAP in comparison to the control, while there was no significant change in the total cellular protein content of the SAP-treated 3D multilayer compared to the control (Fig. 3A).

Treatment with SAP resulted in a 5.3- and 5.9-fold increase in the collagen level in the $2 \mathrm{D}$ monolayer and the $3 \mathrm{D}$ multilayer, re- spectively, (Fig. 3B). However, when the amount of collagen was normalized to the total protein amount, the increase in the level of collagen after SAP treatment in the 3D multilayer was clearly greater (ca. 1.8-fold) than in the 2D monolayer, being a 6.1- and 3.3 -fold increased level in the 3D multilayer and 2D monolayer, respectively, (Fig. 3C). The amount of pro-collagen I alpha 1 in the cell culture medium of the control and SAP-treated 2D monolayers and 3D multilayers showed no statistically significant difference (Fig. S4 ${ }^{1}$ ).

Next, large-sized 3D-DFs were produced in a 6-well plate. An intact sheet of 3D-DFs could be peeled off the culture plate, indicating that the $3 \mathrm{D}-\mathrm{DF}$ sheets are quite robust (Fig. S5 ${ }^{1}$ ). The 3D-DF sheets contracted after being harvested, resulting in a thicker structure.

\subsection{Decellularization and recellularization of 3D-DFs}

Confocal images revealed that the ECM of decellularized 3D-DFs retained its structure, as shown by type I collagen staining (Fig. 4A,B). No nucleus staining was observed, indicating the successful removal of nuclei (Fig. 4A-C). The decellularized $3 \mathrm{D}-\mathrm{DF}$ was reseeded with DFs at a $5 \mathrm{~L}$ cell density (recellularized). An intact multilayered structure of recellularized 3D-DF was obtained after 1 day of culture without addition of SAP (Fig. 4D). In contrast, a multilayered tissue could not be obtained when the DFs were seeded on a blank membrane (Fig. 4E).

\subsection{Gap closure assay of the 3D-DFs}

To investigate the potential use of 3D-DFs for wound healing, a gap closure assay was performed. A wound was made on $3 \mathrm{D}-\mathrm{DF}$ s by puncturing them with a $1-\mathrm{mm}$ punch. The inner edge of the wounded 3D-DFs contracted soon after the puncture wound was made, resulting in a larger wound than the original punched area (Fig. 5A). The wounded area appeared thick- 

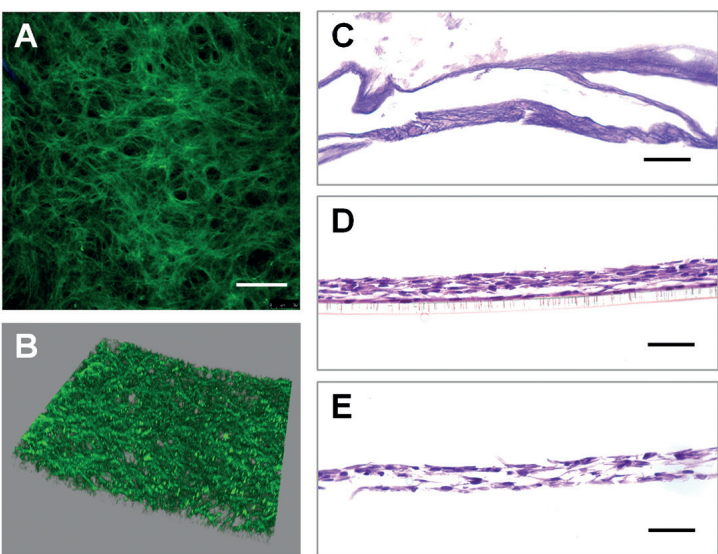

E

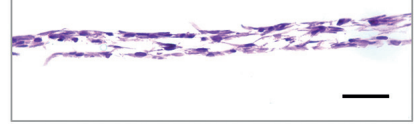

Fig. 4: ECM scaffold from decellularized 3D-DFs

3D-DFs were constructed at $7 L$ density for 3 days in the presence of $0.1 \mathrm{mM}$ SAP before decellularization. (A) Confocal image of the decellularized 3D-DF stained with type I collagen (green) and DAPI (blue). No stained nuclei were observed after the decellularization process. (B) 3D-reconstructed image of (A). (C) The decellularized 3D-DF. (D) The decellularized 3D-DF re-seeded with a $5 \mathrm{~L}$ cell density of DFs and cultured in the absence of SAP for 1 day. (E) DFs were seeded on a blank membrane at a $5 \mathrm{~L}$ cell density and cultured in the absence of SAP for 1 day as control. Scale bars are $100 \mu \mathrm{m}$ (A) and $50 \mu \mathrm{m}$ (C-E).
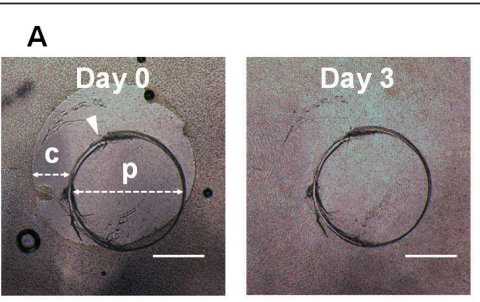

B
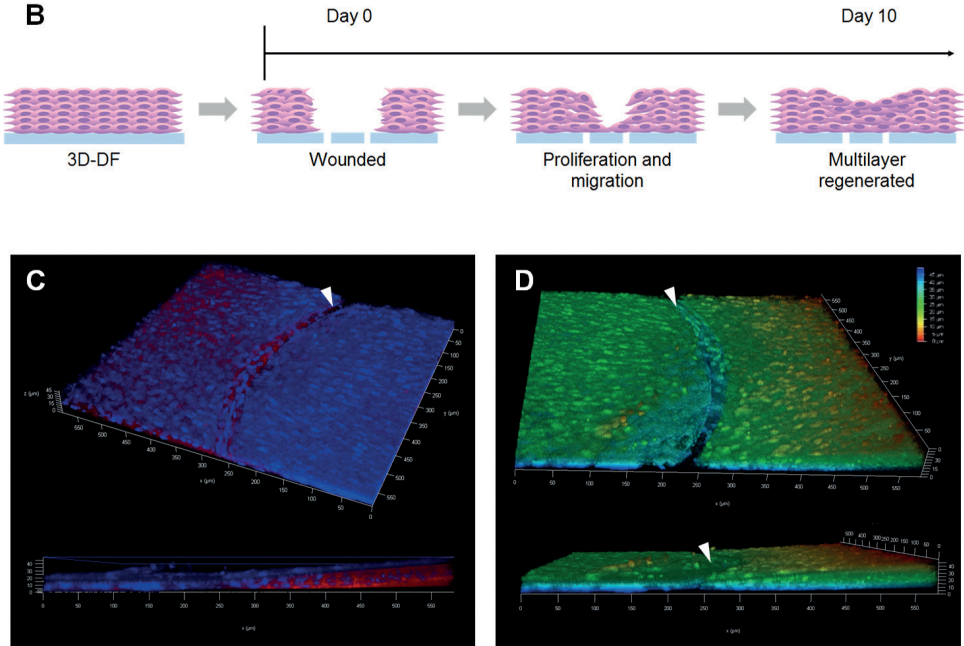

Fig. 5: Assessment of 3D-DFs in a gap closure assay

(A) Phase-contrast images of 3D-DFs 0, 3, 7 and 10 days after wounding with a $1 \mathrm{~mm}$-punch. Scale bars are $500 \mu \mathrm{m} ; \mathrm{p}$ and c refer to the punched area and the area left by tissue contraction, respectively. (B) Schematic illustration of the regeneration of the multilayered structure of the 3D-DF after the wounding. (C) 3D reconstructed CLSM image of the 3D-DF 10 days after wounding. Cells are stained with phalloidin (red). Nuclei are stained with DAPI (blue). (D) Representative CLSM image showing a depth profile of (C). White arrows indicate the incision on the membrane. Cells were found to proliferate and migrate across the incisional space on the cut membrane and formed a multilayered structure. er with cells as time progressed, as shown in phase contrast images (Fig. 5B), indicating that the DFs proliferate and migrate from the original 3D-DF to the wounded area. Seven days after wounding, the wounded area was completely filled with DFs and appeared no different from the unwounded area. The CLSM images revealed that the multilayered structure of 3D-DFs was renewed, as a stacking of several nuclei was evident in the wounded area. The DFs were even found in the incision of the membrane (Fig. 5C,D). Positive staining of Ki-67 was found in the wounded area (Fig. S6 ${ }^{1}$ ), indicating that cells were proliferating during the gap closure process.

\subsection{D-DF grafting on wounded human in vitro skin models}

Dermis grafting was demonstrated in vitro using FTMs. The dermis and the epidermis at the center of the FTMs were removed after punching (Fig. 6A and Fig. S7 ${ }^{1}$ ). The collagen gel of the FTMs contracted slightly after being punched, leaving a space on the membrane that was larger than the original punched area. The wounded areas were seeding with DFs or a 3D-DF graft was inserted onto the membrane below the wounded area (Fig. S7C ${ }^{1}$ ). In the control experiment (no DFs or 3D-DF added), cells were found in the wounded area (Fig. 6B, E, H, K); however, this was 


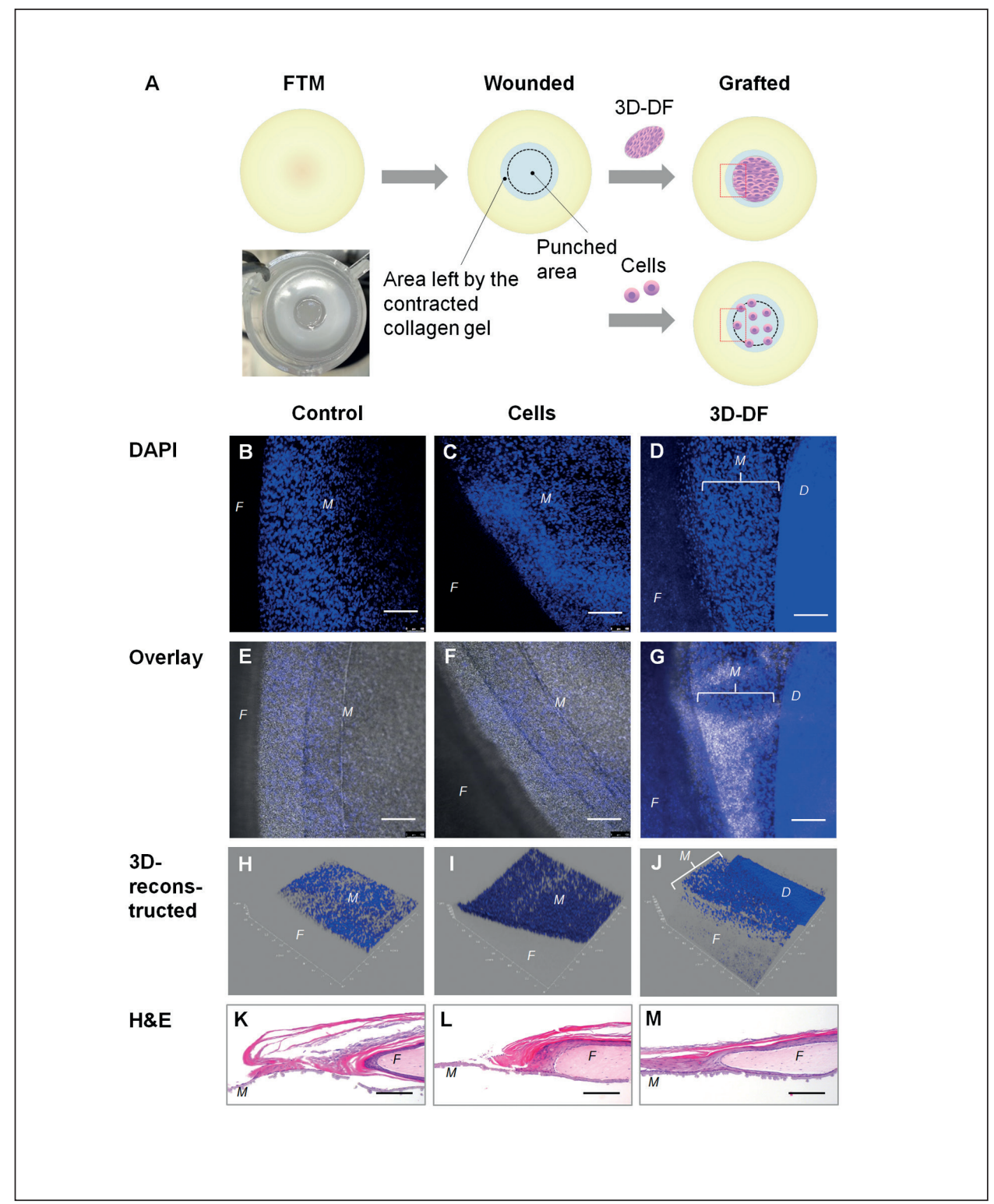

Fig. 6: Dermis graft of 3D-DFs on wounded in vitro human FTMs

(A) Schematic illustration of the grafting experiment. After punching, a 3D-DF graft was placed into the membrane at the site of FTM removal (inset photo), or the site was seeded with fibroblasts. (B-J) Representative CLSM images of FTMs 14 days after wounding. (K-M) Representative H\&E stained cross-sectional images. The wound $(\mathrm{B}, \mathrm{E}, \mathrm{H}, \mathrm{K})$ without grafting or cell seeding (control), (C, F, I, L) seeded with fibroblasts and $(D, G, J, M)$ grafted with the 3D-DF. Images show the interface between the edge of the FTM and the membrane area from which the FTM was removed (red dashed box in A). F, FTM; $M$, membrane, $D$, 3D-DFs. All scale bars are $250 \mu \mathrm{m}$.

only a monolayer. Seeding with DFs showed no difference to the control experiment (Fig. 6C, F, I, L). However, tissue bridging was found between the 3D-DF graft and the FTM, as evidenced in CLSM images that showed stacked nuclei forming a multilayer-like structure (Fig. 6D, G, J, M) and tissue protrusion from the FTM (Fig. 6M). The formation of tissue bridging indicates that the 3D-DF was successfully grafted to the wounded FTM and enhanced the wound healing.

\section{Discussion}

In current methods for the construction of multilayered fibroblasts it takes a long time for the fibroblasts to proliferate and accumulate ECM. Since cells lose their ECM and adhesion proteins upon enzyme (e.g., trypsin) treatment during the detach- ment process, they cannot attach to each other. Therefore, the cells cannot be seeded at a density above the confluent density to form a 3D structure unless given an ECM or ECM components coated to the cell surface to promote cell-cell interactions (Nishiguchi et al., 2011).

In the present study, by seeding DFs at a confluency higher than that of the monolayer and culturing them in the presence of SAP, a 3D-DF structure was formed within 3 days. The seeded DFs were stacked into a multilayered tissue and could be harvested as an intact sheet of DF tissue, which cannot be achieved when cultured in the absence of SAP.

However, after 7 days the 3D-DFs began to lose density (Fig. $\mathrm{S}^{1}$ ) and expression of fibrillin-1, a microfibril component, and ECM glycoproteins seemed to decrease from day 3 (Fig. S8 ${ }^{1}$ ), suggesting disintegration of ECM components over a longer culture time. 
The accelerated cellular self-assembly method enables construction of multilayered DF tissues by seeding cells at up to a $10 \mathrm{~L}$ cell density (approximately $50 \mu \mathrm{m}$ in thickness). To achieve this, we seeded a second layer onto the constructed tissue to obtain a double thickness tissue layer (Fig. S2 ${ }^{1}$ ). However, this method results in poorer tissue structure and needs to be further improved.

The presence of ascorbic acid is known to increase the collagen production in fibroblasts. We investigated the effect of the multilayer structure of DFs on SAP-induced collagen production. Distinctly higher levels of collagen were produced in the $3 \mathrm{D}$ multilayer compared to the 2D monolayer although the total protein concentration in the $3 \mathrm{D}$ multilayer remained constant (Fig. 3). It was not surprising that the total protein of the $2 \mathrm{D}$ monolayer increased when treated with SAP, since ascorbic acid also stimulates cell proliferation (Yamamoto et al., 1992; Phillips et al., 1994). However, the total protein content of the control and SAP-treated 3D multilayer showed no significant difference (Fig. 3A). The higher cell density of the 3D multilayer probably inhibited cell proliferation due to contact inhibition of fibroblasts (Guelstein et al., 1973; Takai et al., 2008).

It was previously found that the amount of DNA in 3D multilayers of fibroblasts constructed by a layer-by-layer technique remained stable during 4 weeks of culture, while that in fibroblast monolayers continued to increase (Chetprayoon et al., 2013). Moreover, the rate of total protein synthesis declines when the cells are in a quiescent state (Aumailley et al., 1982; Fuge et al., 1994; Chen et al., 2012). The collagen level in the SAP-treated 2D monolayer was not significantly greater than in the control 2D monolayer when normalized to the total protein level. However, the ability of SAP to stimulate collagen production was drastically increased when the DFs were in a 3D multilayer although total protein synthesis remained constant. This is consistent with a previous report that the synthesis of collagen as a proportion of the total protein level increases as the culture becomes confluent or the cell density increases (Aumailley et al., 1982). In addition, fibroblasts cultured in a 3D structure might have improved cellular functions and thus be better stimulated by SAP. Unlike a monolayer, where cells are cultured on a rigid surface, cells grown in a 3D environment are surrounded by other cells and intercellular matrices and so have increased cellcell and cell-matrix interactions, leading to improved cellular functions that better reflect those of in vivo tissues (Petrie and Yamada, 2012; Edmondson et al., 2014; Htwe et al., 2015). Such a phenomenon was also observed in the increased elastin synthesis in fibroblasts cultured in $3 \mathrm{D}$ gels as compared to $2 \mathrm{D}$ cultures (Derricks et al., 2013).

Even though it was expected that the DFs in the 3D multilayer would not be proliferating, the 3D-DF multilayer could be regenerated after wounding, and evidence of cell proliferation was found in the wounded area (Fig. 5 and $\mathrm{S}^{1}$ ). In contrast, the DF monolayers do not form a multilayer, even after having been cultured for a month (Chetprayoon et al., 2013). Unlike the DF monolayer, the DFs in the 3D structure in this study proliferated and migrated into the wounded area to form a multilayer without the aid of SAP (Fig. 5B). We have to note that the 3D-DFs represent only a part of the in vivo wound healing system since they are composed of only fibroblasts. Other cell types, such as immune cells or a vascular network, may be introduced to the $3 \mathrm{D}-\mathrm{DF}$ in future studies to better represent in vivo wound healing. Nevertheless, the 3D-DFs can potentially be a useful in vitro 3D model of gap closure during the wound healing process to study the efficacy of drugs or other compounds.

The constructed 3D-DF was decellularized to obtain an ECM scaffold. The resulting ECM showed a 3D meshwork of collagen that can serve as a scaffold for culturing other cells. To test the ability of the decellularized 3D-DF to support cell growth, fresh DFs were seeded onto ECM scaffolds at an over-confluent density. Multilayered DFs were obtained after 1 day of culture. A cell-derived matrix has an advantage over a synthetic matrix due to its better biocompatibility and its containing components that regulate cellular functions, including cell attachment, proliferation, migration and differentiation, as in the native tissue environment (Theocharis et al., 2016). Multilayered fibroblasts cultured by current methods require a long culture time, typically of more than several weeks, before they are ready to be decellularized to obtain the ECM (Scherzer et al., 2015; Xing et al., 2015; Franco-Barraza et al., 2017). The accelerated cellular self-assembly described in this study can produce ECM scaffolds within 3 days, which will be useful for various applications.

The use of the 3D-DF for skin grafting was demonstrated using human in vitro FTMs (Fig. 6) as an alternative to an animal experiment (Fig. 6). Wounded FTMs grafted with 3D-DF sections showed enhanced re-epithelialization, while seeding cells into the wounded area failed to result in wound closure, possibly due to an inadequate supporting matrix for the skin cells to migrate and regenerate. Moreover, seeding of single cells requires time for the cells to attach to the area, resulting in loss of some cells before they can attach. This is similar to in vivo situations, where cell therapy by injection of cells fails to deliver the cells to target sites unless biomaterials are used for cell transport (Suzuki et al., 2018). We demonstrate that the 3D-DF can be grafted onto the wounded dermis in vitro. The 3D-DF provides a supporting matrix for the FTM to re-epithelialize, especially type I collagen, which facilitates the migration of keratinocytes along the dermal matrix (Rousselle et al., 2019). Our results suggest that the 3D-DF may be suitable for use as a skin substitute for wound treatment. Since the 3D-DF can be constructed within a few days, it can be readily supplied for the urgent needs of skin grafts in wound treatment.

\section{Conclusions}

We present a simple concept for the fast construction of scaffold-free 3D tissues by cellular self-assembly. The process was accelerated by seeding DFs at an over-confluent density and culturing them with SAP as a collagen stimulant, enabling the formation of 3D-DFs within a few days. The synergistic effect of ascorbic acid and the 3D structure on stimulating collagen production was found to be key to achieving the 3D-DFs. ECM could be derived from the 3D-DFs and was suitable as a scaf- 
fold for 3D cell culture. Furthermore, the 3D-DFs had the ability to regenerate into a multilayered tissue after wounding, suggesting a useful in vitro model for wound closure studies, and may be suitable as dermal grafts for wound treatments. The presented technique may be applicable to other cell types for construction of $3 \mathrm{D}$ tissues.

\section{References}

Alaribe, F. N., Manoto, S. L. and Motaung, S. C. K. M. (2016). Scaffolds from biomaterials: Advantages and limitations in bone and tissue engineering. Biologia 71, 353-366. doi:10.1515/biolog-2016-0056

Anderson, J. M., Rodriguez, A. and Chang, D. T. (2008). Foreign body reaction to biomaterials. Semin Immunol 20, 86-100. doi:10.1016/j.smim.2007.11.004

Aumailley, M., Krieg, T., Razaka, G. et al. (1982). Influence of cell density on collagen biosynthesis in fibroblast cultures. Biochem J 206, 505-510. doi:10.1042/bj2060505

Barnes, M. J. (1975). Function of ascorbic acid in collagen metabolism. Ann N Y Acad Sci 258, 264-277. doi:10.1111/ j.1749-6632.1975.tb29287.x

Bokhari, M., Carnachan, R. J., Cameron, N. R. et al. (2007). Culture of HepG2 liver cells on three dimensional polystyrene scaffolds enhances cell structure and function during toxicological challenge. J Anat 211, 567-576. doi:10.1111/j.1469-7580. 2007.00778.x

Chen, B. R., Cheng, H. H., Lin, W. C. et al. (2012). Quiescent fibroblasts are more active in mounting robust inflammatory responses than proliferative fibroblasts. PLoS One 7, e49232. doi:10.1371/journal.pone.0049232

Chetprayoon, P., Kadowaki, K., Matsusaki, M. et al. (2013). Survival and structural evaluations of three-dimensional tissues fabricated by the hierarchical cell manipulation technique. Acta Biomater 9, 4698-4706. doi:10.1016/j.actbio. 2012.08.019

Chung, J. H., Youn, S. H., Kwon, O. S. et al. (1997). Regulations of collagen synthesis by ascorbic acid, transforming growth factor-beta and interferon-gamma in human dermal fibroblasts cultured in three-dimensional collagen gel are photoaging- and aging-independent. J Dermatol Sci 15, 188-200. doi:10.1016/ S0923-1811(97)00607-5

Derricks, K. E., Rich, C. B., Buczek-Thomas, J. A. et al. (2013). Ascorbate enhances elastin synthesis in 3D tissue-engineered pulmonary fibroblasts constructs. Tissue Cell 45, 253-260. doi:10.1016/j.tice.2013.03.001

Dondossola, E., Holzapfel, B. M., Alexander, S. et al. (2017). Examination of the foreign body response to biomaterials by nonlinear intravital microscopy. Nat Biomed Eng 1, 1-20. doi:10.1038/s41551-016-0007

Edmondson, R., Broglie, J. J., Adcock, A. F. et al. (2014). Three-dimensional cell culture systems and their applications in drug discovery and cell-based biosensors. Assay Drug Dev Technol 12, 207-218. doi:10.1089/adt.2014.573

Franco-Barraza, J., Beacham, D. A., Amatangelo, M. D. et al. (2017). Preparation of extracellular matrices produced by cultured and primary fibroblasts. Curr Protoc Cell Biol 71, 10.9.1-10.9.34. doi:10.1002/cpcb.2

Fuge, E. K., Braun, E. L. and Werner-Washburne, M. (1994). Protein synthesis in long-term stationary-phase cultures of Saccharomyces cerevisiae. J Bacteriol 176, 5802-5813. doi:10. 1128/jb.176.18.5802-5813.1994

Geesin, J. C., Darr, D., Kaufman, R. et al. (1988). Ascorbic acid specifically increases type I and type III procollagen messenger RNA levels in human skin fibroblast. J Invest Dermatol 90, 420-424. doi:10.1111/1523-1747.ep12460849

Gerullis, H., Georgas, E., Borós, M. et al. (2014). Inflammatory reaction as determinant of foreign body reaction is an early and susceptible event after mesh implantation. Biomed Res Int 2014, 1-6. doi:10.1155/2014/510807

Gribova, V., Liu, C. Y., Nishiguchi, A. et al. (2016). Construction and myogenic differentiation of 3D myoblast tissues fabricated by fibronectin-gelatin nanofilm coating. Biochem Biophys Res Commun 474, 515-521. doi:10.1016/j. bbrc.2016.04.130

Griffith, L. G. and Swartz, M. A. (2006). Capturing complex 3D tissue physiology in vitro. Nat Rev Mol Cell Biol 7, 211-224. doi: $10.1038 / \mathrm{nrm} 1858$

Guelstein, V. I., Ivanova, O. Y., Margolis, L. B. et al. (1973). Contact inhibition of movement in the cultures of transformed cells. Proc Natl Acad Sci U S A 70, 2011-2014. doi:10.1073/ pnas.70.7.2011

Hata, R. and Senoo, H. (1989). L-ascorbic acid 2-phosphate stimulates collagen accumulation, cell proliferation, and formation of a three-dimensional tissuelike substance by skin fibroblasts. J Cell Physiol 138, 8-16. doi:10.1002/jcp.1041380103

Htwe, S. S., Harrington, H., Knox, A. et al. (2015). Investigating NF-KB signaling in lung fibroblasts in 2D and 3D culture systems. Respir Res 16, 1-9. doi:10.1186/s12931-015-0302-7

Jean, J., Lapointe, M., Soucy, J. et al. (2009). Development of an in vitro psoriatic skin model by tissue engineering. J Dermatol Sci 53, 19-25. doi:10.1016/j.jdermsci.2008.07.009

Jean, J., Garcia-Pérez, M. E. and Pouliot, R. (2011). Bioengineered skin: The self-assembly approach. J Tissue Sci Eng S5, 001. doi:10.4172/2157-7552.S5-001

Kim, S. H., Lee, H. R., Yu, S. J. et al. (2015). Hydrogel-laden paper scaffold system for origami-based tissue engineering. Proc Natl Acad Sci U S A 112, 15426-15431. doi:10.1073/ pnas. 1504745112

Knight, E. and Przyborski, S. (2015). Advances in 3D cell culture technologies enabling tissue-like structures to be created in vitro. J Anat 227, 746-756. doi:10.1111/joa.12257

L'Heureux, N. Pâquet, S., Labbé, R. et al. (1998). A completely biological tissue-engineered human blood vessel. FASEB J 12, 47-56. doi:10.1096/fasebj.12.1.47

Lee, J. H., Lee, H., Joung, Y. K. et al. (2011). The use of low molecular weight heparin-pluronic nanogels to impede liver fibrosis by inhibition the TGF- $\beta /$ Smad signaling pathway. Biomaterials 32, 1438-1445. doi:10.1016/j.biomaterials.2010. 10.023

Lee, W., Debasitis, J. C., Lee, V. K. et al. (2009). Multi-layered culture of human skin fibroblasts and keratinocytes through 
three-dimensional freeform fabrication. Biomaterials 30, 1587-1595. doi:10.1016/j.biomaterials.2008.12.009

Loh, Q. L. and Choong, C. (2013). Three-dimensional scaffolds for tissue engineering applications : Role of porosity and pore size. Tissue Eng Part B Rev 19, 485-502. doi:10.1089/ten. TEB.2012.0437

Michel, M., L'Heureux, N., Pouliot, R. et al. (1999). Characterization of a new tissue-enineered human skin equivalent with hair. In Vitro Cell Dev Biol Anim 35, 318-326. doi:10.1007/ s11626-999-0081-x

Murad, S., Tajima, S., Johnson, G. R. et al. (1983). Collagen synthesis in cultured human skin fibroblasts: Effect of ascorbic acid and its analogs. J Invest Dermatol 81, 158-162. doi:10. 1111/1523-1747.ep12543573

Nishiguchi, A., Yoshida, H., Matsusaki, M. et al. (2011). Rapid construction of three-dimensional multilayered tissues with endothelial tube networks by the cell-accumulation technique. Adv Mater 23, 3506-3510. doi:10.1002/adma.201101787

Ohgoda, O., Sakai, A., Koga, H. et al. (1998). Fibroblast-migration in a wound model of ascorbic acid-supplemented threedimensional culture system: The effects of cytokines and malotilate, a new wound healing stimulant, on cell-migration. J Dermatol Sci 17, 123-131. doi:10.1016/S0923-1811 (98)00003-6

Onoe, H., Okitsu, T., Itou, A. et al. (2013). Metre-long cell-laden microfibres exhibit tissue morphologies and functions. Nat Mater 12, 584-590. doi:10.1038/nmat3606

Peck, M., Dusserre, N., McAllister, T. N. et al. (2011). Tissue engineering by self-assembly. Mater Today 14, 218-224. doi:10.1016/S1369-7021(11)70117-1

Petrie, R. J. and Yamada, K. M. (2012). At the leading edge of three-dimensional cell migration. J Cell Sci 125, 5917-5926. doi: $10.1242 /$ jcs.093732

Phillips, C. L., Combs, S. B. and Pinnell, S. R. (1994). Effects of ascorbic acid on proliferation and collagen synthesis in relation to the donor age of human dermal fibroblasts. J Invest Dermatol 103, 228-232. doi:10.1111/1523-1747.ep12393187

Ramanujan, S., Pluen, A., McKee, T. D. et al. (2002). Diffusion and convection in collagen gels: Implications for transport in the tumor interstitium. Biophys $J$ 83, 1650-1660. doi:10.1016/ S0006-3495(02)73933-7
Rousselle, P., Montmasson, M. and Garnier, C. (2019). Extracellular matrix contribution to skin wound re-epithelialization. Matrix Biol 75-76, 12-26. doi:10.1016/j.matbio.2018.01.002

Scherzer, M. T., Waigel, S., Donninger, H. et al. (2015). Fibroblast-derived extracellular matrices: An alternative cell culture system that increases metastatic cellular properties. PLoS One 10, 1-17. doi:10.1371/journal.pone.0138065

Suzuki, G., F. Young, R. and Harue, S. (2018). Where are cellbased therapies heading? Current limitations and future directions. Hear Res Open J 4, 71-77. doi:10.17140/hroj-4-144

Takai, Y., Miyoshi, J., Ikeda, W. et al. (2008). Nectins and nectin-like molecules: Roles in contact inhibition of cell movement and proliferation. Nat Rev Mol Cell Biol 9, 603-615. doi: $10.1038 / \mathrm{nrm} 2457$

Theocharis, A. D., Skandalis, S. S., Gialeli, C. et al. (2016). Extracellular matrix structure. Adv Drug Deliv Rev 97, 4-27. doi:10. 1016/j.addr.2015.11.001

Xing, Q., Yates, K., Tahtinen, M. et al. (2015). Decellularization of fibroblast cell sheets for natural extracellular matrix scaffold preparation. Tissue Eng Part C Methods 21, 77-87. doi:10.1089/ten.tec.2013.0666

Yamamoto, I., Muto, N., Murakami, K. et al. (1992). Collagen synthesis in human skin fibroblasts is stimulated by a stable form of ascorbate, 2-O-alpha-D-glucopyranosyl-L-ascorbic acid. J Nutr 122, 871-877. doi:10.1093/jn/122.4.871

\section{Conflict of interest}

The authors declare that they have no conflicts of interest.

\section{Acknowledgements}

We would like to thank to Ms Chotip Phooim (Histology Unit, Department of Pathology, Faculty of Medicine, Siriraj Hospital, Mahidol University) for histological sections. We appreciate Mr Robert Butcher (Chulalongkorn University) for editing this manuscript. This work was supported by the Research, Development and Engineering Fund through the National Nanotechnology Center (NANOTEC), NSTDA, Thailand. 